\title{
Adaptation of Conduit Artery Vascular Smooth Muscle Tone to Induced Hypertension
}

\author{
P. Fridez, ${ }^{1}$ A. Makino, ${ }^{2}$ D. Kakol, ${ }^{2}$ H. MiYazaki, ${ }^{2}$ J.-J. Meister, ${ }^{3}$ K. HAyashi, ${ }^{2}$ and N. Stergiopulos ${ }^{1}$ \\ ${ }^{1}$ Laboratory of Hemodynamics and Cardiovascular Technology, Swiss Federal Institute of Technology, Lausanne, Switzerland; \\ ${ }^{2}$ Department of Systems and Human Science, Division Mechanical Science, Graduate School of Engineering Science, Osaka \\ University, Japan; and ${ }^{3}$ Laboratory of Biomechanics and Biophysics of Cells, Swiss Federal Institute of Technology, \\ Lausanne, Switzerland
}

(Received 13 August 2001; accepted 17 May 2002)

\begin{abstract}
We studied the changes in vascular smooth muscle (VSM) cell tone during the adaptation of rat common carotids to induced hypertension. Hypertension was induced in 8 week old male Wistar rats by total ligation of the aorta between the two kidneys. Mean blood pressure increased abruptly from 92 $\pm 2 \mathrm{~mm} \mathrm{Hg}($ mean $\pm \mathrm{SE})$ to $145 \pm 4 \mathrm{~mm} \mathrm{Hg}$ and remained constant thereafter. Rats were sacrificed 2, 4, 8, and 56 days after surgery and the left common carotid artery was excised for analysis. Pressure-diameter curves were measured in vitro under normal, maximally contracted, and totally relaxed VSM. The VSM tone was analyzed in terms of its basal tone (active stress at low strains) and its myogenic tone (increase in active stress at high strains). Our results show that the capacity of the VSM to develop maximal active stress is not altered in hypertension. Basal tone, however, increases rapidly in the acute hypertension phase (2-8 days postsurgery) and drops to nearly control values at 56 days postsurgery. Also, the onset of myogenic response decreases to lower strains following the step change in pressure, to be restored back to control levels at 56 days postsurgery. We conclude that VSM adaptation is most significant in the acute hypertension phase and acts as a first, rapid defense mechanism for the arterial wall. The VSM tone returns back to normal levels once the slower geometrical and structural remodeling is developed sufficiently to restore the biomechanical environment and function of the arterial wall to control levels. (C) 2002 Biomedical Engineering Society.
\end{abstract}

[DOI: $10.1114 / 1.1507326]$

Keywords-Arterial wall, Remodeling, Adaptation, Myogenic response, Smooth muscle tone, VSM, Aortic banding, Conduit vessel.

\section{INTRODUCTION}

The adaptation of the arterial wall to induced hypertension has been studied in great detail in conduit arteries ${ }^{5,6,13,16,24,26}$ as well as in resistance arteries and arterioles. ${ }^{1,4,12,15,30}$ In conduit arteries, the focus was on the structural and morphological remodeling of the arte-

Address correspondence to Nikos Stergiopulos, Professor, Laboratory of Hemodynamics and Cardiovascular Technology, Swiss Federal Institute of Technology, 1015 Lausanne, Switzerland. Electronic mail: nikolaos.stergiopulos@epfl.ch rial wall, and there was little attention paid to the changes in vascular smooth muscle (VSM) tone, with the exception of the case of spontaneously hypertensive rats (SHR). ${ }^{2,3,20,21}$ In contrast with the studies on conduit arteries, alterations of VSM tone in resistance vessels exposed to hypertension have been extensively studied. ${ }^{8,17,27}$

The lack of interest in VSM tone remodeling in conduit vessels exposed to hypertension is likely to be related to the minor importance accorded to the VSM tone in regulating conduit artery function and to the ambiguities on physiological significance of the myogenic response in large vessels. Yet, conduit arteries may exhibit significant spontaneous tone ${ }^{7}$ and, depending on the artery, may have a large myogenic contraction capacity. ${ }^{10,25}$

It is well recognized that conduit arteries use VSM tone to adapt their lumen diameter to changes in flow (shear stress-dependent mechanism) ${ }^{19}$ or to pressure (myogenic mechanism). ${ }^{10,25}$ It is likely that the same mechanisms are involved in the acute (hours) or early phase (first few days) of arterial wall remodeling in response to a step change in pressure. However, how the VSM tone develops if the step change in pressure is sustained over longer periods (days to weeks) remains unknown.

The purpose of this study is to obtain a complete tracing of VSM tone adaptation in conduit arteries subjected to a sustained step change in pressure. We examine how VSM tone adapts immediately after the pressure rise and how it remodels itself in the long run. We focus not only on the maximal contractile capacity of the VSM but also on normal VSM tone (often termed resting or residual VSM tone), because this reflects the physiological state of VSM and is thus most indicative of the functional adaptation of the vascular smooth muscle to sustained hypertension. 


\section{MATERIALS AND METHODS}

\section{In Vivo Experimental Procedures}

The details of the experimental procedures and experimental results used in this paper for tone analysis were already published and explained in detail in a previous publication $^{9}$ and will only be briefly summarized here.

Hypertension was induced in male Wistar rats, aged 56-63 days (8 weeks) and weighing $270-312 \mathrm{~g}$, by total ligation of the aorta between the two kidneys according to the procedure described in Michel et al. ${ }^{11}$

\section{Arterial Preparations}

Experiments were terminated 2, 4, 8, and 56 days postsurgery ( $n=6$ for each group). Two control groups were included for the measurement of arterial properties in normotensive rats. One was the presurgery control group (day 0 control) and the other one was age matched to the 56 days postsurgery group (day 56 control; $n=6$ for each). We have not included a sham operated group at 8 days since the acute part of these experiments last only 1 week which is a small period in comparison with the age of the animals ( 8 weeks). Thus, the biomechanical properties of the control group would not change substantially within this small period of time. As described in a previous publication, ${ }^{9}$ the left common carotid (LCC) artery was carefully exposed, marked, and measured lengthwise to determine the in vivo axial extension ratio. The rats were then killed and the LCC was excised. A segment obtained from the proximal end of the LCC was used for mechanical testing and the remaining segment was used to measure geometrical properties. All mechanical tests were finished within $9 \mathrm{~h}$ postmortem and the specimens were kept in KrebsRinger solution during this period (at $5{ }^{\circ} \mathrm{C}$ for storage and slowly warmed up to $37^{\circ} \mathrm{C}$ for measurements).

\section{Biomechanical Measurements and Analysis}

Pressure-Diameter Curves. The preparations and the measurement methods used in this study for the inflation/ extension tests were published in a separate paper. ${ }^{9}$ The excised LCC segment was inflated and deflated at a rate of approximately $170 \mathrm{~Pa} / \mathrm{s}(1.3 \mathrm{~mm} \mathrm{Hg} / \mathrm{s})$. The external diameter and the intraluminal pressure (under no flow conditions) were measured. Arteries were preconditioned by means of 4-6 inflation-deflation cycles (pressure range $0-26.7 \mathrm{kPa}$, i.e., $0-200 \mathrm{~mm} \mathrm{Hg}$ and rate of approximately $170 \mathrm{~Pa} / \mathrm{s}$, i.e., $1.3 \mathrm{~mm} \mathrm{Hg} / \mathrm{s}$ ) until the pressure-diameter $(p-d)$ curve became reproducible. The ascending limb of the last stable loop was recorded and used for the data analysis under normal VSM tone conditions. The $p-d$ curves under maximal contraction were obtained using NE $(5 \times 10-7 \mathrm{~mol} / \mathrm{L})$ without pre- conditioning. Finally, the $p-d$ curves under total relaxation were obtained using Papaverine $(10-4 \mathrm{~mol} / \mathrm{L})$ with preconditioning.

Geometrical Analysis. Four rings were cut from the middle part of the long distal LCC segment and kept for imaging following the procedures described in a previous paper. ${ }^{9}$ Then these images were used to determine the internal radius, $R_{i}$, and the thickness, $H$, and these in turn were used to calculate the wall cross-sectional area at zero load, WCSA $=\pi\left(2 R_{i} H+H^{2}\right)$. Finally, assuming that the wall is incompressible and using the formulas: $\lambda_{z} \pi\left(r_{0}^{2}-r_{i}^{2}\right)=$ WCSA and $h=\left(r_{0}-r_{i}\right)$, where $\lambda_{z}$ is the in situ axial extension ratio, the deformed internal radius, $r_{i}$, and deformed wall thickness, $h$, are calculated at any given pressure.

Stress Analysis. The mean circumferencial wall stress, $\sigma_{\text {tot }}$, was calculated using Laplace's law

$$
\sigma_{\mathrm{tot}}=\frac{P_{i} r_{i}}{r_{0}-r_{i}}
$$

The mean circumferencial wall stretch ratio, $\lambda$, indicative of the stretch of the midwall fiber was calculated as follows:

$$
\lambda=\frac{r_{0}+r_{i}}{R_{0}+R_{i}}
$$

For simplicity, the mean circumferencial wall stretch ratio $\lambda$ will shortly be referred to as "strain."

Based on the classic Hill model, ${ }^{16}$ the mean circumferential stress (hoop stress) can be written as a sum of its active and passive components

$$
\sigma_{\text {tot }}=\sigma_{\text {pas }}+\sigma_{\text {act }} .
$$

The active component of the hoop stress, $\sigma_{\text {act }}$, accounts for the stress borne by the VSM, and therefore will be used as a biomechanical surrogate for VSM tone. The passive component, $\sigma_{\text {pas }}$, accounts for the stress borne by the "passive" components of the wall (i.e., extracellular matrix, VSM in completely relaxed state, etc.). In Hill's model, the active and passive stress components are assumed to be independent. Thus, the effect of VSMextracellular matrix coupling is neglected in this approach.

This study focuses on VSM tone and thus, on the characterization of the active stress $\sigma_{\text {act }}$. Figure 1 shows how the active stress is assessed. In Fig. 1(A), we see a typical set of $p-d$ curves of the rat common carotid artery obtained under total VSM relaxation (solid line), 
A

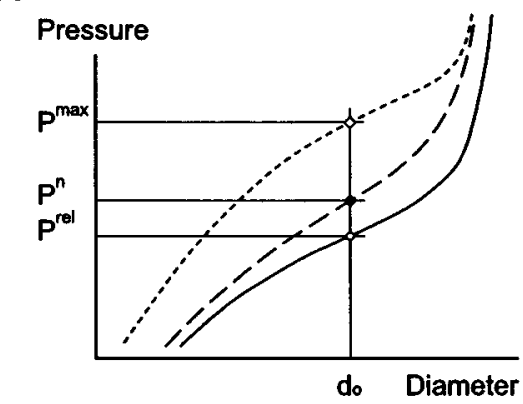

B

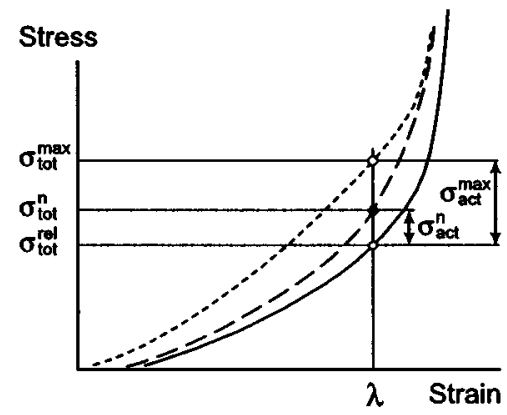

C

Active

Stress

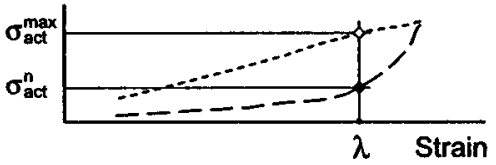

D

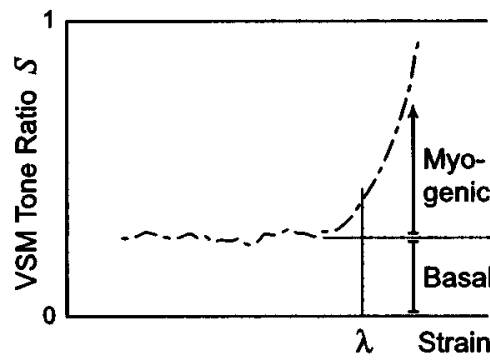

_- VSM under Total Relaxation
_ - VSM under Normal Conditions
_...- VSM under Maximal Contraction

FIGURE 1. (A) Typical pressure-diameter curves from a common carotid belonging to an 8 day hypertension group rat. Data were measured in vitro under conditions of total relaxation (solid line), under normal VSM tone (dashed line) and under maximal contraction (dotted line) of the vessel. (B) Transformation of the pressure-diameter curves of (A) to the stress-strain domain according to Eq. (4). (C) Active stress-strain curves obtained from (B) by subtracting passive stress from total stress under normal VSM tone (dashed line) and from total stress under maximal contraction (dotted line) [Eqs. (5) and (6)]. (D) Dependence of the VSM tone ratio on strain. VSM tone ratio is obtained from (C) and Eq. (8). The two separate domains, the first one being the region where basal tone determines the total VSM tone (low strain values, horizontal branch of the curve) and the second domain being the region where the myogenic tone dominate the total VSM tone (higher strain values, increasing branch of the curve) are seen.

normal VSM tone (dashed line), and maximal VSM contraction (dotted line). For a given wall distension (diameter $\left.d_{0}\right)$, the total stress under total relaxation $\left(\sigma_{\text {tot }}^{\mathrm{rel}}\right)$, the total stress under normal VSM tone $\left(\sigma_{\text {tot }}^{n}\right)$, and the total stress under maximal VSM tone $\left(\sigma_{\text {tot }}^{\max }\right)$ are calculated using Laplace's law

$$
\sigma_{\text {tot }}^{\mathrm{rel}}=\frac{P^{\mathrm{rel}} r}{h}, \quad \sigma_{\text {tot }}^{n}=\frac{P^{n} r}{h}, \quad \text { and } \quad \sigma_{\text {tot }}^{\max }=\frac{P^{\mathrm{max}} r}{h} \text {, }
$$

where $r$ and $h$ are the internal radius and wall thickness corresponding to the external diameter $d_{0}$, respectively. The superscripts on stress denote the state of VSM tone ( $n=$ normal tone; $\max =$ maximal contraction; $r e l=$ total relaxation). The definitions of the passive and total stresses given earlier lead to a stress-strain relationship depicted in Fig. 1(B). The subscripts refer to the stress components according to Hill's model (tot=total, pas $=$ passive, act $=$ active $)$. Obviously, $\sigma_{\text {tot }}^{\text {rel }}=\sigma_{\text {pas }}$. Subtracting the passive stress from the total stress we obtain the active stress under normal VSM tone

$$
\sigma_{\mathrm{act}}^{n}=\sigma_{\mathrm{tot}}^{n}-\sigma_{\mathrm{pas}}
$$

and under maximal VSM contraction

$$
\sigma_{\mathrm{act}}^{\max }=\sigma_{\text {tot }}^{\max }-\sigma_{\text {pas }} .
$$

Typical active stress-strain curves are shown in Fig. $1(\mathrm{C})$.

\section{Mathematical Description of Active Stress}

To facilitate the analysis of VSM tone development in hypertension, we use a simple mathematical model for the description of active stress. As seen in Fig. 1(C) (see also the Results section), active stress under maximal contraction $\left(\sigma_{\mathrm{act}}^{\max }\right)$ is a linear function of the strain, $\lambda$, and therefore we assume it has the following form:

$$
\sigma_{\mathrm{act}}^{\max }(\lambda)=c_{0}+c_{1} \lambda
$$

Equation (7) is limited to the ascending portion of the active stress-strain curve, which can be approximated quite well by a straight line. Normally, the active stress at maximal contraction is not a straight line, but a function with a descending part at higher strains, such as a 
bell-shape curve, or a parabolic curve, as been suggested by other authors. ${ }^{4}$ For the sake of simplicity, we chose to simply model the ascending part of the curve with a straight line and not speculate on the form of the curve at higher strains.

For a given level of strain, $\lambda$, and a given level of VSM tone, the smooth muscle develops an active stress, $\sigma_{\text {act }}^{n}$, which is in the range $0 \leqslant \sigma_{\text {act }}^{n} \leqslant \sigma_{\text {act }}^{\max }$. For a given strain $\lambda$, we define the VSM tone ratio $S(\lambda)$ as the ratio between the active stress under normal tone and the active stress under maximal contraction

$$
S(\lambda)=\frac{\sigma_{\text {act }}^{n}(\lambda)}{\sigma_{\text {act }}^{\max }(\lambda)}, \quad 0 \leqslant S(\lambda) \leqslant 1 .
$$

Thus, the nondimensional parameter $S$ characterizes the degree of VSM tone, $S=0$ meaning no tone and $S=1$ meaning maximal contraction.

Figure 1(D) shows the VSM tone ratio, $S(\lambda)$, curve obtained using the data in Fig. 1(C) and Eq. (8). Therefore, the $S(\lambda)$ curve in Fig. 1(D) depicts the relative contraction of vascular smooth muscle as a function of the strain under normal VSM tone conditions. We observe that the relative degree of VSM contraction exhibits two different domains of behavior. In the first domain, at low strain range, $S(\lambda)$ remains shallow and fairly constant, we refer to this part of the $S(\lambda)$ curve as the "basal tone." However, in the second domain, at higher strain values, the VSM tone ratio, $S(\lambda)$, increases rapidly. This represents a strain-activated increase in VSM tone which is characteristic of the myogenic mechanism and this part of $S(\lambda)$ will therefore be referred to as the "myogenic tone." Based on the characteristics of the graph in Fig. 1(D), we assume that the behavior of the VSM tone ratio in these two distinct domains can be accounted for by a sum of two functions $\left[S_{\text {bas }}, S_{\text {myo }}(\lambda)\right]$ depicting the basal and the myogenic tone, respectively, as follows:

$$
S(\lambda)=S_{\text {bas }}+S_{\text {myo }}(\lambda) .
$$

$S_{\text {bas }}$ is independent of the strain $\lambda$ and will be referred to as the basal tone ratio and $S_{\text {myo }}(\lambda)$ will be referred to as the myogenic tone ratio. As on full strain range, the active stress under normal tone tends to behave as a sigmoid (see also Fig. 4 in the Results section), and the myogenic tone ratio accounts for the inflection domain of the sigmoid curve, we assume it has the following form:

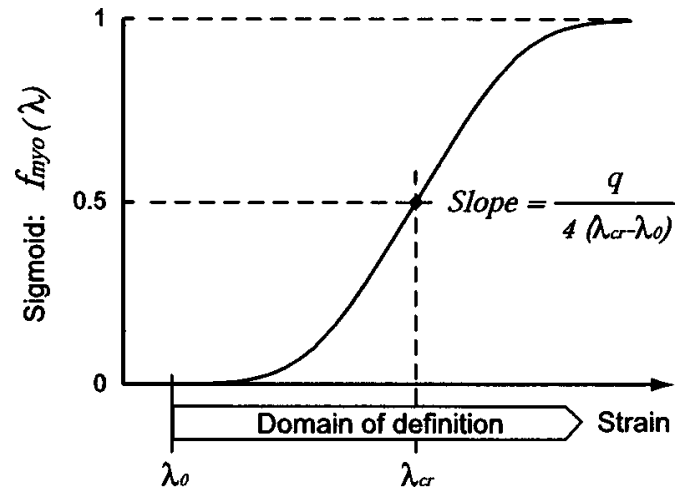

FIGURE 2. Shape of the sigmoid function $\left[f_{\text {myo }}(\lambda)\right]$ myogenic activation. $q$ is proportional to the slope of the sigmoid at the inflection point, $\lambda_{\mathrm{cr}}$ is the strain at the inflection point (myogenic critical strain), and $\lambda_{0}$ is the relative length of the circumferencial midwall fiber at zero pressure.

$$
\begin{aligned}
S_{\text {myo }}(\lambda) & =\left(1-S_{\text {basal }}\right) f_{\text {myo }}(\lambda) \\
& =\left(1-S_{\text {basal }}\right) \frac{\left(\frac{\lambda-\lambda_{0}}{\lambda_{\mathrm{cr}}-\lambda_{0}}\right)^{q}}{1+\left(\frac{\lambda-\lambda_{0}}{\lambda_{\mathrm{cr}}-\lambda_{0}}\right)^{q}},
\end{aligned}
$$

where $q \lambda_{\mathrm{cr}}$, and $\lambda_{0}$ are the parameters of the sigmoid (see Fig. 2). $\lambda_{\text {cr }}$ is the strain at the inflection point of the sigmoid, $f_{\text {myo }}(\lambda)$, and it can be viewed as the middle point of the range of $\lambda$ values comprising the myogenic response. Thus, we will refer to $\lambda_{\text {cr }}$ as the myogenic critical strain. The parameter $q$ is proportional to the maximal slope of the VSM tone ratio as a function of strain (i.e., proportional to the slope at the inflection point, Fig. 2). The parameter $\lambda_{0}$ is the relative length of the circumferencial midwall fiber at zero pressure and thus is entirely determined by the experimental geometry: the arterial segments are stretched longitudinally and this causes the length of the circumferencial mid wall fiber to contract to $\lambda_{0}$. The factor $\left(1-S_{\text {bas }}\right)$ in the definition of $S_{\text {myo }}(\lambda)$ accounts for the fact that the myogenic tone can only operate on the residual tone capacity of the VSM, which is the tone remaining between total contraction $(S=1)$ and the basal tone ratio $\left(S_{\mathrm{bas}}\right)$.

This description leads to the following representation of active stress under normal VSM tone:

$$
\sigma_{\text {act }}^{n}(\lambda)=\sigma_{\text {act }}^{\max }(\lambda) S(\lambda)
$$

with $\sigma_{\text {act }}^{\max }$ and $S(\lambda)$ given by Eqs. (5), (7), (8), and (10).

The parameters of model equations derived above leading to a mathematical description of the active stress are determined through fitting the experimental data. This leads to a description of the short- and long-term evolution of VSM tone in response to induced hyperten- 

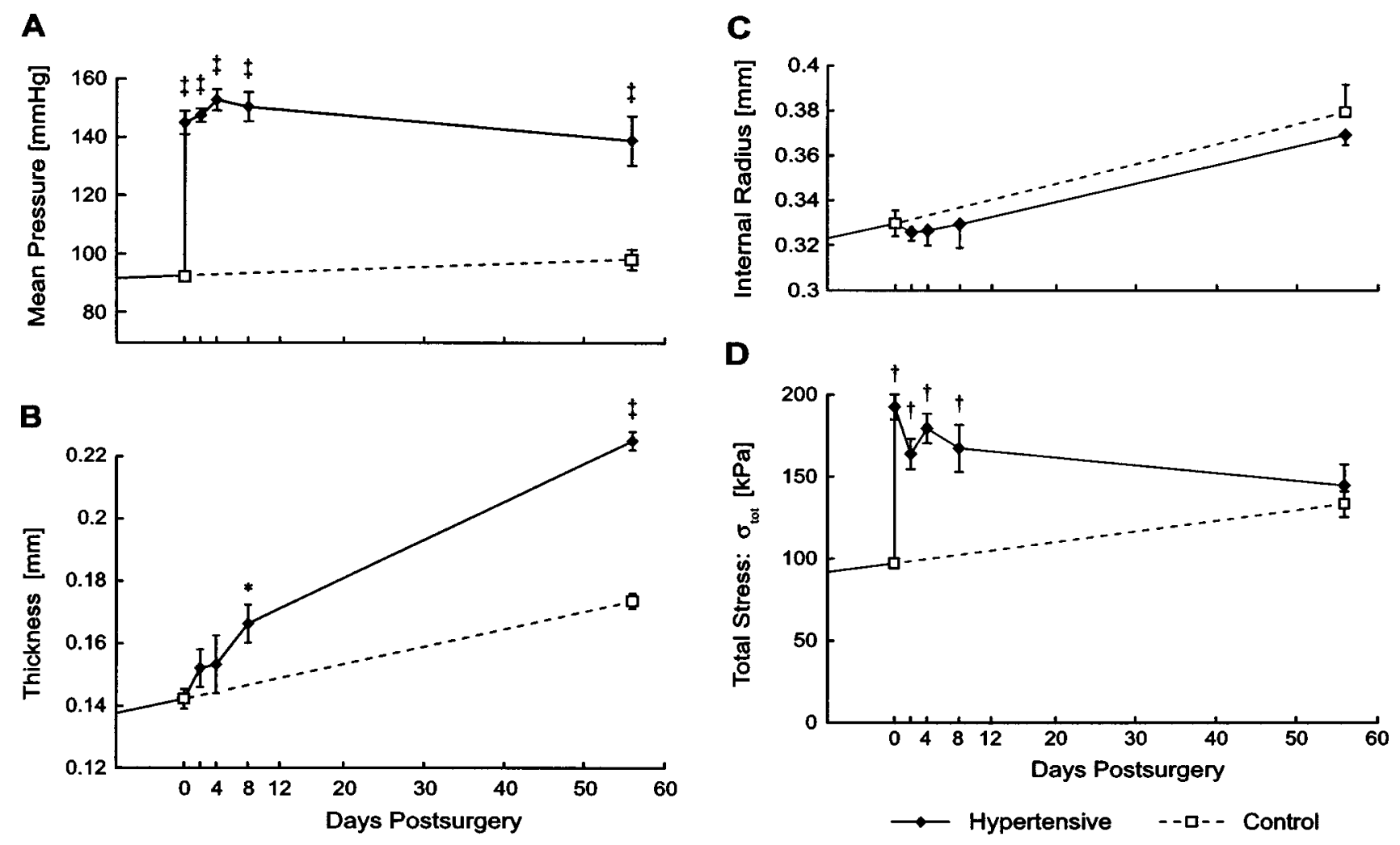

FIGURE 3. (A) Evolution of aortic mean pressure. Error bars indicate $S E$ ( $n=25,22,16,12,6,6$, from left to right, respectively). (B) Evolution of wall thickness at zero load state $(n=6$, for each point). (C) Evolution of the internal radius at zero load state $(n=6)$. (D) Evolution of mean wall stress at mean pressure $(n=6)$. In all figures the dashed lines (open squares) and the solid lines (black diamonds) show the evolution of the control and hypertensive groups, respectively. The character $\neq$ indicates $p$ $<0.005$ with respect to control at day 0 and day 56 ; $\dagger$ indicates $p<0.005$ with respect to control at day 0 ; and $*$ indicates $p$ $<0.05$ with respect to control at day 0 .

sion. Then, the evolution of VSM tone is assessed in terms of its maximal contractile capacity (parameters $c_{0}$, $\left.c_{1}\right)$, basal tone ratio $\left(S_{\text {bas }}\right)$, and myogenic tone ratio (parameters $\lambda_{\mathrm{cr}}$ and $q$ ).

\section{Numerical and Statistical Analysis}

The five parameters of the model $\left(c_{0}, c_{1}, S_{\text {basal }}, \lambda_{\text {cr }}\right.$, and $q$ ) were determined using a standard LevenbergMarquardt method (least square fit).

All data were expressed as mean \pm SE. Comparisons between each group and the control ( 0 days postsurgery) were made with student's $t$ test. Differences were considered to be significant for $p<0.05$.

\section{RESULTS}

Following aortic ligation mean blood pressure increased abruptly from $12.3 \pm 0.3 \mathrm{kPa}$ (mean $\pm \mathrm{SE}$ ) to $19.3 \pm 0.5 \mathrm{kPa}(92 \pm 2$ and $145 \pm 4 \mathrm{~mm} \mathrm{Hg}$, respectively) and remained constant for the first week postsurgery. At 56 days postsurgery, it had slightly diminished to 18.4 $\pm 1.2 \mathrm{kPa}(138 \pm 9 \mathrm{~mm} \mathrm{Hg})$ [Fig. 3(A)]. The experimen- tal results on which the stress-strain calculations are based were explained in detail in a previous publication ${ }^{9}$ and will only be briefly described here.

\section{Geometrical Adaptation}

A gradual increase of the wall thickness at no load state corresponding to $118 \% \pm 4 \%$ of the control value at 8 days postsurgery, and $140 \% \pm 2 \%$ of the control value at 56 days postsurgery was observed [Fig. 3(B)]. This increase in thickness with respect to control (day 0) found at 8 and 56 days postsurgery was statistically significant $(p<0.05)$. The changes in the internal radius at mean pressure were not significant during the entire postsurgery period [Fig. 3(C)]. Therefore, we have an eccentric hypertrophy, as typically reported in the literature ${ }^{6,22}$ (hypertrophic which could not be classified as "inward" nor "outward" remodeling). ${ }^{33}$

\section{Time Evolution of Stress-Strain Curves}

The evolution of mean wall stress is shown in Fig. 3(D). Figure 3 shows the stress-strain curves for the 
A

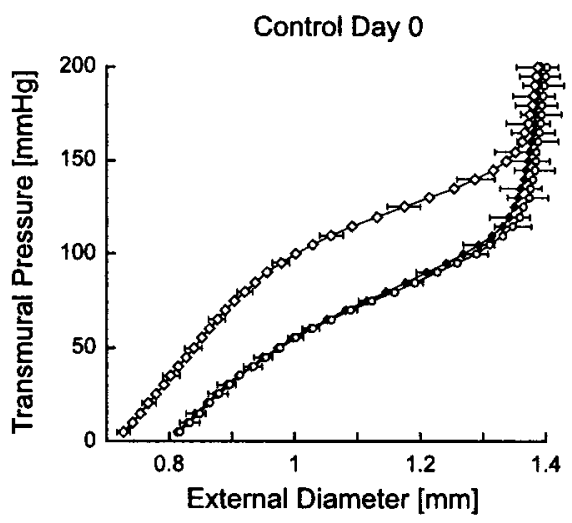

D

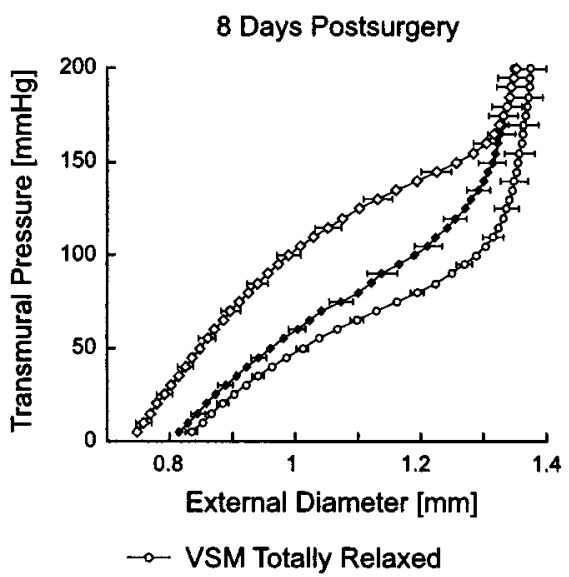

B

2 Days Postsurgery

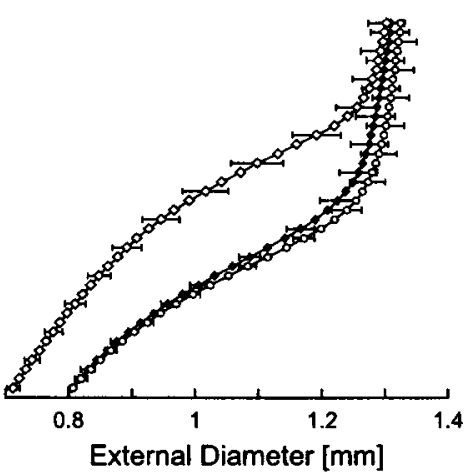

E

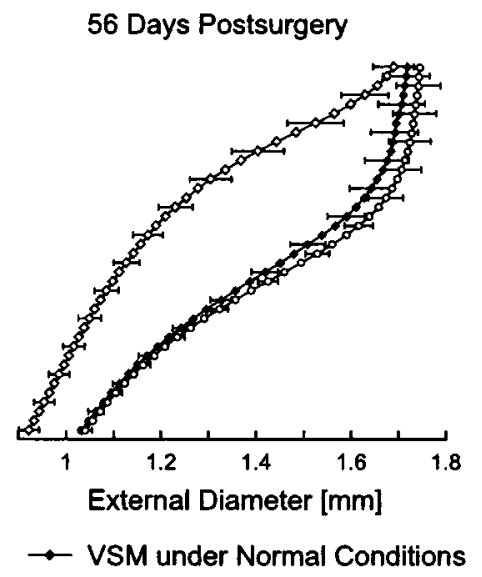

C

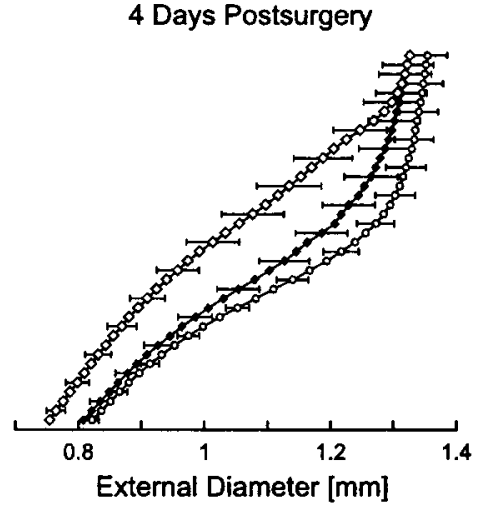

$\mathbf{F}$

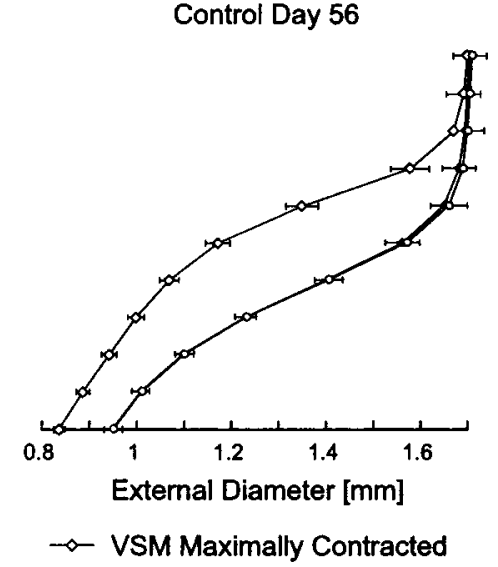

FIGURE 4. Pressure-diameter curves under normal VSM tone ( $P^{n}$, squares), under maximally contracted VSM ( $P^{\text {max }}$, open diamonds) and under totally relaxed VSM ( $P^{\text {rel }}$, filled circles). (A) and $(F)$ show the results for the control groups at days 0 and 56. (B), (C), (D), and (E) show data for hypertension groups at 2, 4, 8, and 56 days postsurgery, respectively. Measurements were performed at in situ length. Error bars indicate SE $(n=6)$.

control group at day 0 , the hypertensive groups at $2,4,8$, and 56 days postsurgery as well as the age-matched control group at day 56. The three curves in each graph represent the stress-strain relation under normal VSM tone (black diamonds), under maximal contraction (diamonds), and under total relaxation (circles).

For the control groups, we observe that the stressstrain curve under normal VSM tone lies very close to the stress-strain curve under total relaxation. This means that under normotensive conditions, the rat common carotid exhibits very little VSM tone. We may thus conclude that in the normotensive rat, under physiological conditions, the arterial properties are almost entirely determined by the passive components of the wall. However, immediately after exposure to high blood pressure, we observe a significant development in active stress under normal VSM tone. This is clearly seen at 4 and 8 days postsurgery [Figs. 3(C) and 3(D)], where the stressstrain under normal VSM tone (black diamonds) "de- parts" from a position close to the stress-strain curve under total relaxation (circles) and moves to the middle of the operating stress-strain domain. At 56 days postsurgery [Fig. 3(E)], however, active stress under normal VSM tone seems to regress and the stress-strain curve under normal conditions moves back to the stress-strain curve under total relaxation.

\section{Adaptation of VSM Tone}

The adaptation of the VSM tone to hypertension is seen in Fig. 4(A) which shows the evolution of the active components of the stress under normal VSM tone $\left[\sigma_{\text {act }}^{n}\right.$, Eq. (5)] and under maximal contraction $\left[\sigma_{\text {act }}^{\max }\right.$, Eq. (6)]. Regarding the active stress under maximal contraction, two points are worth noting: first, it is seen that the maximal active stress, $\sigma_{\text {act }}^{\max }$ [thick lines, Fig. 4(A)], is linearly proportional to the strain, $\lambda$, for the main part $(0-24 \mathrm{kPa}$ or $0-180 \mathrm{~mm} \mathrm{Hg})$ of the tested pressures 
range $(0-26.7 \mathrm{kPa}$ or $0-200 \mathrm{~mm} \mathrm{Hg})$. This is depicted by the linear relationship between $\sigma_{\text {act }}^{\max }$ and $\lambda$ presented in the Methods section [Eq. (7)]. Second and most important, all $\sigma_{\text {act }}^{\max }$ curves are similar for $\lambda$ values less than 1.3 , which is the mean working condition under maximal contraction. This similarity implies that the capacity of the VSM to develop maximal active stress is insensitive to hypertension.

In contrast with the active stress under maximal contraction, which remains unchanged, hypertension leads to profound changes in the active stress under normal VSM tone. This is clearly seen in Fig. 4(A) (thin lines) where we observe an important increase in basal tone immediately after the onset of hypertension (increase in slope of the initial quasilinear portion of the curves). This increase continues until 8 days postsurgery. At 56 days postsurgery, however, basal tone returns back near the control levels. The myogenic tone also changes very rapidly in presence of hypertension: the high slope portion of the curves shift towards the left, which signifies a reduction of the myogenic critical strain $\left(\lambda_{\mathrm{cr}}\right)$ as part of the VSM adaptation to hypertension. This means that the myogenic response takes place at lower strain (earlier) in presence of hypertension. Again, as for the basal tone, the changes in $\lambda_{\mathrm{cr}}$ are transitory, that is, at long term (56 days postsurgery), $\lambda_{\text {cr }}$ returns back near the control values. There are no significant differences in the slopes of the upstroke curves (right portion of the thin lines) characterizing the myogenic response. This suggests that, as a first approximation, the slope parameter $q$ (see Fig. 2) can be considered constant.

Black diamonds in Fig. 4(A) indicate the operating point corresponding to the in vivo mean pressure and normal VSM tone. We clearly see that the basal tone does indeed reflect the state of VSM tone for the control group (0 and 56 days). In that sense the somewhat arbitrary term basal tone used in the present paper is justified. Figure 4(A) shows also that with induced hypertension the artery operates under higher pressure and increased stretch. At this state the myogenic mechanism takes part by increasing tone, and therefore the artery is no longer at the basal tone state.

\section{Modeling and Parameter Estimation}

To obtain a quantitative description of the adaptation of the VSM tone in response to hypertension, we fitted the simple theoretical model describing active stress under maximal contraction [Eq. (7)] and under normal VSM tone [Eqs. (9), (10), and (11)] to the experimental data.

The active stress under maximal contraction does not appear to change significantly in hypertension [Fig. 4(A)]. Moreover, the mean in vivo strain for maximally contracted curves is less than 1.3 (i.e., near the region where the curves are more similar). Thus, we used a single function [Eq. (7)] fit to the entire data set [thick lines, Fig. 4(A)] and obtained the thick solid line in Fig. 4(B). The parameters of the fit are $c_{0}=-44.8(\mathrm{kPa})$ and $c_{1}=56.6(\mathrm{kPa})$ [Eq. (7)].

The model equation for the active stress under normal VSM tone [Eq. (11)] was fitted to experimental results [thin lines, Fig. 4(A)] in order to obtain the time evolution of parameters $S_{\mathrm{bas}}$ and $\lambda_{\mathrm{cr}}$. Because the slope of the myogenic response does not vary much during adaptation, we assume that the slope parameter $q$ is the same for all groups. To determine its value, we used the data obtained at 2, 4, 8, and 56 days postsurgery and control at day 0 and day 56 [six curves shown in Fig. 4(A) representing normal VSM tone, thin lines]. The obtained $\sigma_{\text {act }}^{n}(\lambda)$ curves are shown in Fig. 4(B) (thin lines).

The evolution of the model parameters, $S_{\text {bas }}$ and $\lambda_{\text {cr }}$, are shown in Figs. 5(A) and 5(B), respectively. Initially, there is a drastic increase in basal tone ratio, $S_{\text {bas }}$, reaching $716 \%$ of the control value at 8 days postsurgery [Fig. $5(\mathrm{~A})]$. This is followed by a gradual decrease near the control value at 56 days postsurgery. We also observe that the myogenic critical strain, $\lambda_{c r}$, undergoes a rapid decrease (by $7.2 \%$ ) during the first 2 days of hypertension followed by a slower but significant decrease (up to $10 \%)$ at 8 days postsurgery compared with the control [Fig. 5(B)]. Thus, similarly to the adaptation of the basal tone ratio, $S_{\text {bas }}$, the myogenic critical strain, $\lambda_{\text {cr }}$, returns to nearly control levels at 56 days postsurgery following a rapid change in the acute hypertension phase. This sheds light on the initial short-term dynamics of the VSM state following induced hypertension, which is qualitatively different than the extensively studied longterm response.

\section{DISCUSSION}

We induced hypertension in 8 week old male Wistar rats by total aortic occlusion, which induces a step increase in pressure, and we monitored the changes in VSM tone of the left common carotid artery. We studied the early phase of the resulting wall adaptation (2-8 days postsurgery) as well as the remodeling at long-term (56 days postsurgery). We found that the early phase of remodeling is characterized by profound changes in the VSM tone, which mainly result from a large-scale adaptation of the basal tone and myogenic response of the VSM cells. In the long term, VSM tone appears to return back to control values, as the arterial wall enters a phase of significant geometrical and morphological adaptation.

The changes in VSM basal tone and myogenic response in relation to arterial wall remodeling in hypertension have been studied extensively in the microcirculation, resistance vessels and in cultured VSM cells. ${ }^{1,15,17,27,28,30,34}$ VSM have also been studied in con- 
A

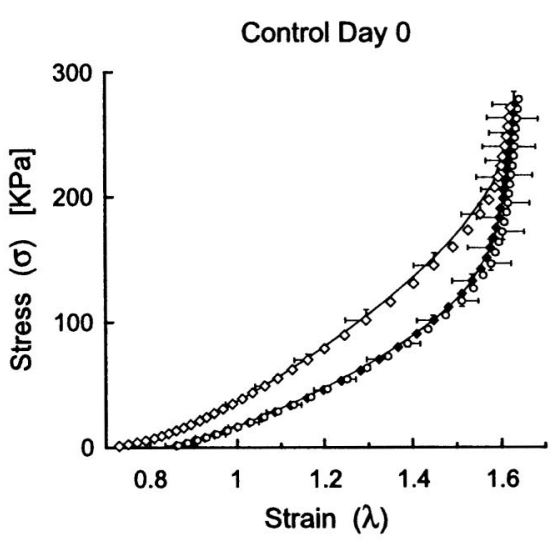

D

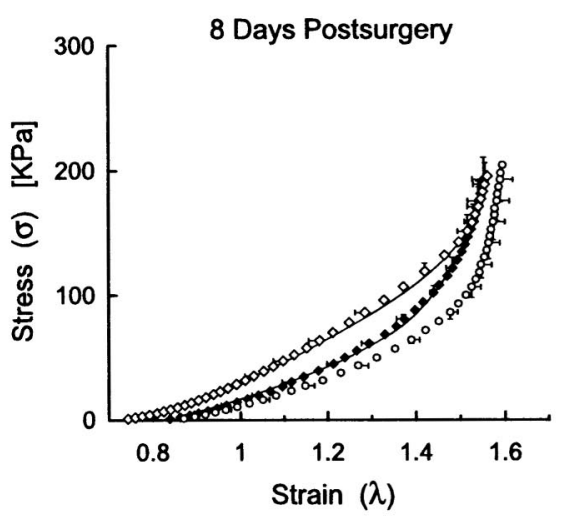

- VSM Totally Relaxed
B

\section{Days Postsurgery}

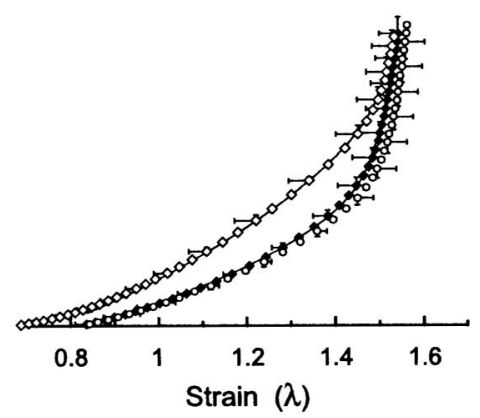

E

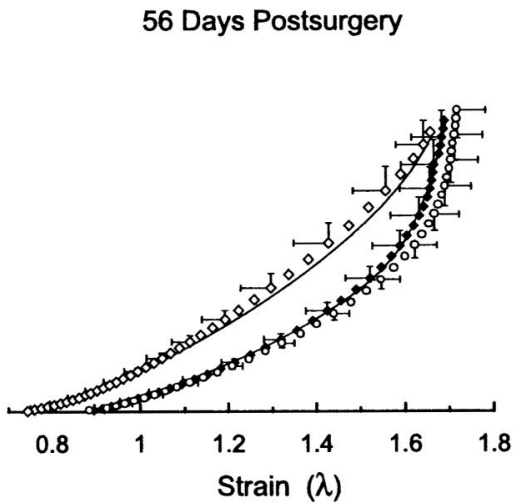

$\diamond$ VSM Maximally Contracted
C

4 Days Postsurgery

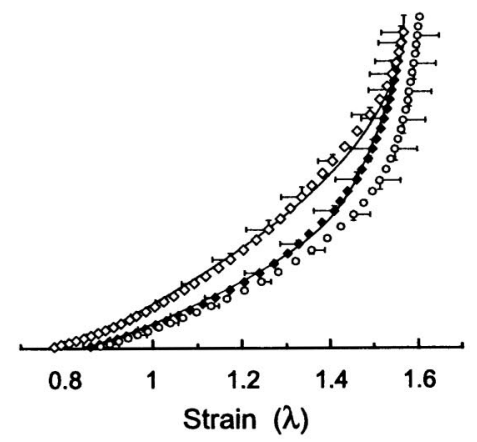

$\mathbf{F}$

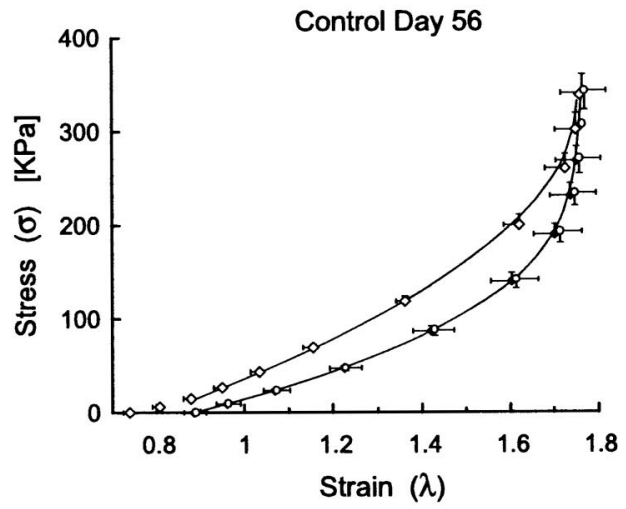

- Results of the model for normal VSM tone and maximally contracted state, respectively

FIGURE 5. Stress-strain curves under normal VSM tone ( $\sigma_{\text {tot }}^{n}$, black diamonds), under maximally contracted VSM ( $\sigma_{\text {tot }}^{\max }$, open diamonds), and under totally relaxed VSM ( $\sigma_{\text {tot }}^{\text {rel }}$, open circles). (A) and (F) show the results for the control groups at days 0 and 56. (B), (C), (D), and (E) show data for groups at 2, 4, 8, and 56 days postsurgery, respectively. Measurements were performed at in situ length. Error bars indicate SE $(n=6)$, and for clarity, error bars are shown at every other two points and in only two directions except in Fig. 3(F) where all error bars are shown for only two directions. The solid lines indicate the result of the theoretical model for normal [Eqs. (9), (10), and (11)] and maximal VSM tone [Eq. (7)].

duit vessels in $\mathrm{SHR}^{20,21,23}$ and in a specific study on rat carotid artery. ${ }^{4}$ The myogenic response of conduit vessels in the pulmonary circulation has also been previously investigated. ${ }^{5}$ The present work is, to our knowledge, the first study in conduit vessels, which shows that acute hypertension leads to profound changes in basal and myogenic VSMC tone. These changes, in turn, contribute considerably to the biomechanical adaptation of the arterial wall.

\section{Acute Versus Long-Term Adaptation}

Arterial wall remodeling in response to acute changes in the mechanical environment is a fairly rapid process. We observed an $18 \% \pm 4 \%$ increase in wall thickness within 8 days after the aortic occlusion, and this mono- tonic increase reached $40 \% \pm 2 \%$ at 56 days postsurgery. It is well recognized that such monotonic increase in wall thickness leads to the restoration of hoop stress to control levels. ${ }^{22}$ Wall remodeling, on the other hand, tends to restore the elastic and structural (compliance) properties of the arterial wall, although this type of structural adaptation takes place at a much slower pace. ${ }^{6}$

From a physiological standpoint, however, both the wall thickening and structural remodeling can be considered as long-term (weeks) and permanent remodeling of the arterial wall to hypertension. Our study shows that the wall adapts its VSM tone at a much faster pace than wall thickening and structural remodeling. The myogenic critical strain which characterizes the onset of the myogenic response undergoes a statistically significant shift from $\lambda_{\mathrm{cr}}=1.64$ (control, day 0 ) to $\lambda_{\mathrm{cr}}=1.47$ (8 days 
A

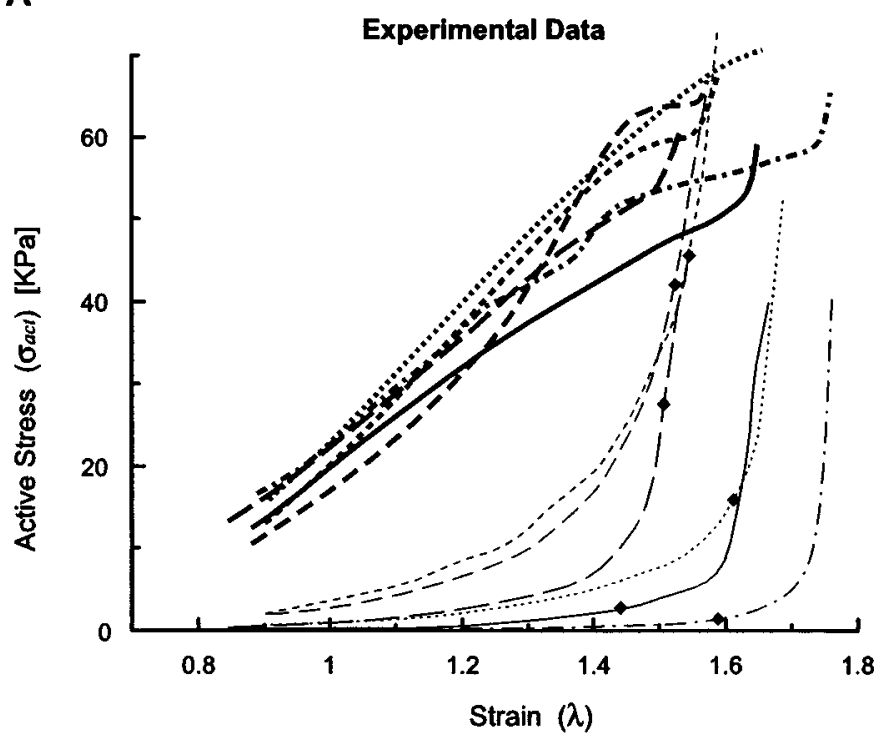

B

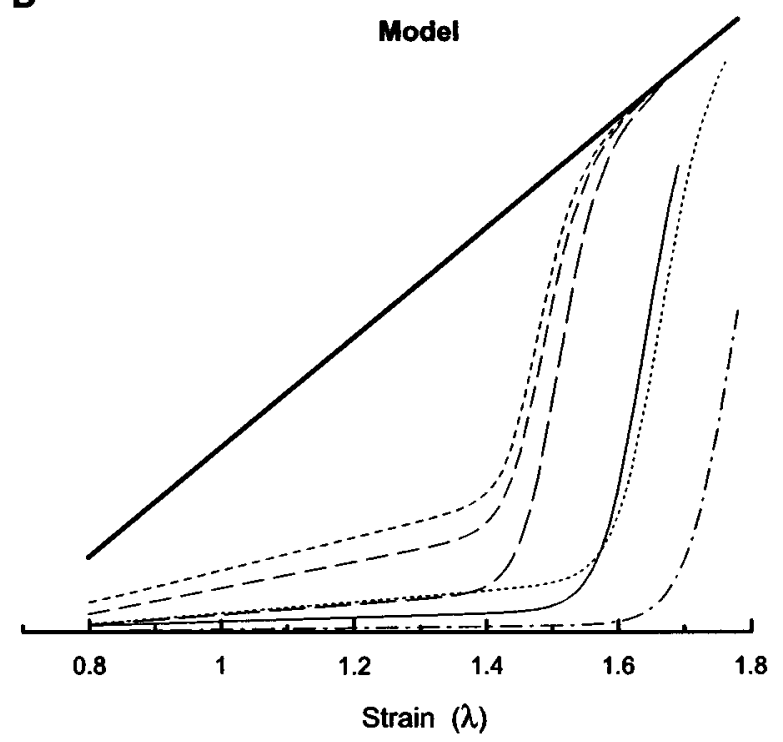

Thick Lines: Active stress under maximal VSM contraction

Thin Lines: Active stress under normal VSM tone

- = Operating point for each rat group, respectively

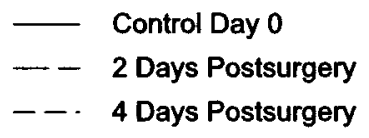

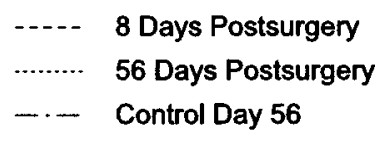

FIGURE 6. (A) Evolution of active stress as a function of strain under maximal contraction (thick lines) and under normal VSM tone (thin lines). Black diamonds indicate the operating point corresponding to the in vivo mean pressure and normal VSM tone. (B) Results of the model fit to curves shown in (A). For active stress under maximal contraction the theoretical result (thick line) is found by fitting the model Eq. (7) through the entire set of experimental curves [control at day 0 and 56, and 2,4,8 and 56 days postsurgery, thick lines in Fig. 4(A)].

postsurgery). In parallel with this, the basal tone ratio exhibits a strong significant increase reaching $527 \%$ of the control value at 4 days, and $716 \%$ of the control value at 8 days postsurgery [Fig. 5(A)]. The time scale of this process indicates that the VSM tone adaptation is the primary defense mechanism that the wall employs to respond to the acute increase in stress and strain.

\section{Physiological Significance of VSM Remodeling}

The means by which the basal tone ratio $\left(S_{\text {bas }}\right)$ is altered and the myogenic mechanism $\left(\lambda_{\mathrm{cr}}\right)$ is displaced to lower distension levels are not known. We observe that for each $\sigma_{\text {act }}^{\max }$ curve a residual myogenic effect exists and corresponds to the myogenic response on the respective $\sigma_{\text {act }}^{n}$ curve. This residual effect is the large increase in slope at maximal strain values of $\sigma_{\mathrm{act}}^{\max }$ curves (thick lines) seen in Fig. 4(A). The value of strain at which this increase occurs coincides with the strain value at which the slope of the corresponding $\sigma_{\text {act }}^{n}$ curve increases (thin lines). This suggests that the strain at which the myogenic mechanism appears $\left(\lambda_{\mathrm{cr}}\right)$ is independent of the amount of basal tone ratio $\left(S_{\mathrm{bas}}\right)$. Thus, the adaptation mechanisms of $\lambda_{\text {cr }}$ and $S_{\text {bal }}$ seem to follow two independent pathways (see Figs. 6 and 7).
The increase in basal tone ratio may eventually be related to changes in the ionic balance of intracellular calcium and/or to an increased sensitivity to intracellular calcium concentration. ${ }^{21}$ The decrease in $\lambda_{\text {cr }}$ represents a displacement of the myogenic response toward lower strain values and conceivably implies a change in membrane stretch activated channel sensitivity or functionality. ${ }^{30}$ However, further research is necessary to elucidate these suggestions.

From a functional standpoint, it seems reasonable to assume that it is faster for the VSM to change its contractile properties, namely the sensitivity and vigor of the working VSM tone, than to synthesize a sufficient amount of ECM scleroproteins for wall thickening and structural remodeling. Therefore, we may hypothesize that, in the acute phase of hypertension (immediately after pressure increase up to a few days), VSM adapts its contractile properties in order to alleviate excessive strain and improve, to some extent, the apparent wall properties (compliance). This takes place while the slower synthetic activity is being established, after which, stress and elastic properties are optimized through wall thickening and structural remodeling.

Our data provides evidence in support of the above hypothesis. In a previous publication ${ }^{9}$ we have shown 
A

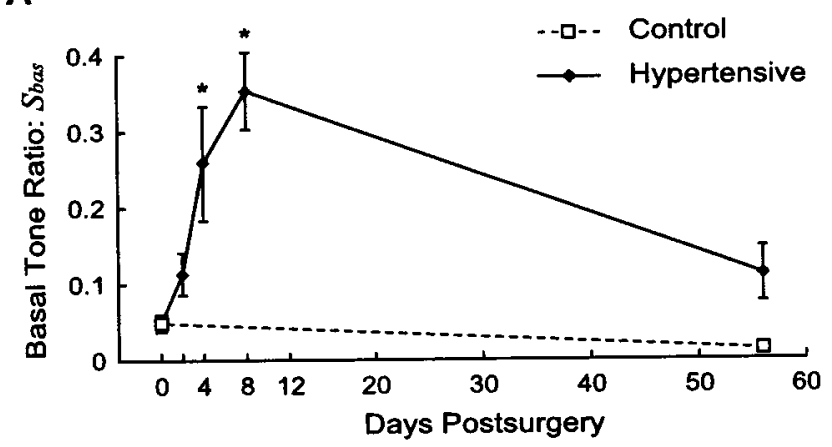

B

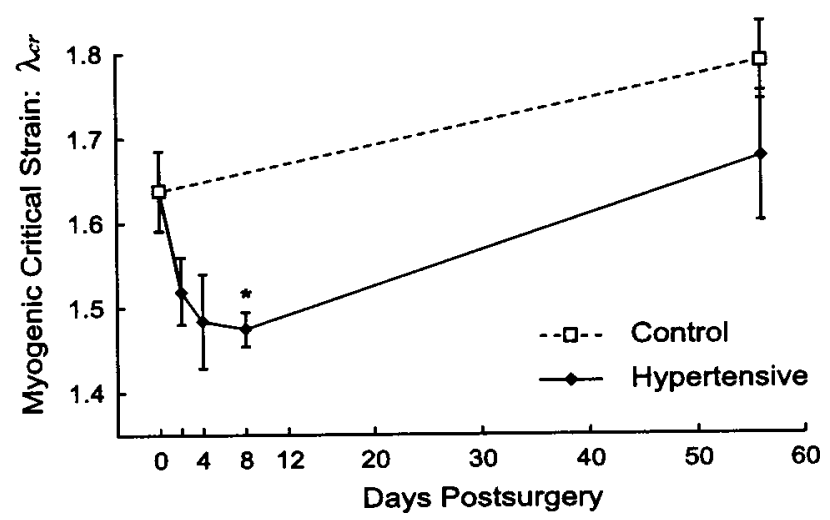

FIGURE 7. Evolution of basal tone ratio, $S_{\text {bas }}(A)$ and myogenic critical strain, $\lambda_{c r}(B)$ in response to hypertension. Bars indicate standard error. Stars indicate statistical significance $(p<0.05)$ with respect to control (at day 0$)$.

that the VSM tone changes lead to a substantial improvement in the apparent structural properties of arterial wall. It was shown that, eight days postsurgery and under normal VSM tone, compliance is significantly larger (by a factor of 1.6) than control at the same operating pressure level under normal VSM tone. However, 8 days postsurgery and under total relaxation (no VSM tone), compliance is not significantly different (larger only by a factor of 0.4) compared with the control at the same operating pressure level under total relaxation (no VSM tone). Thus, VSM response leads to improved conduit artery function by an increase in its buffer (Windkessel) effect.

Our results show that the VSM tone remodeling is a transitory and reversible process. As shown in Fig. 5, both basal tone ratio and myogenic critical strain change significantly during the acute phase (2-8 days postsurgery), and in long term (56 days) their values return near the control levels. However, in the SHR, VSM tone under normal stimulation remains very high on a permanent basis. ${ }^{9,12,21}$ This means that, in the SHR, VSM maintains a considerable amount of permanent contraction throughout the animal's life. Therefore, there are significant dif- ferences in the way VSM is utilized in normotensive and spontaneously hypertensive rats. Thus, simple extrapolation of SHR data on to VSM function in normotensive rats should be exercised with great caution.

\section{Contractile Capacity}

Although basal VSM tone and the myogenic mechanism undergo rapid and significant adaptation during the acute hypertension phase, the capacity of the VSM to develop maximal active stress does not change during the entire hypertension follow up period and remains near control values [Fig. 4(A)]. One possible explanation for this would be that wall thickening and VSM hypertrophy take place in a proportional way. In this case at a given wall section, the ratio of the surface occupied by the VSM to the total surface would remain constant throughout the remodeling period. Since stress is tension (force) per unit area, and the elementary tension developed by each contractile apparatus within the VSM cell is constant, we would expect that the active stress under maximal contraction does not change in hypertension. Further histological measurements may provide support of this hypothesis.

\section{Limitations of the Study}

First, we have performed an in vitro assessment of the elastic properties and VSM tone. It has been reported that the in vitro measurements may differ considerably from in situ and in vivo measurements. ${ }^{29}$

Second, we have neither explicitly tested endothelial function nor included flow in the analysis. Therefore, the influence of endothelium-derived substances on vascular tone and wall properties remains unknown. Previous studies have, nevertheless, shown that in resistance arteries, the basal tone is influenced by pressure and flow independently $y^{3,14,18}$ and that in small arterioles the myogenic response is not modulated by arteriolar blood flow or its attendant shear stress within the physiological range (wall shear rate $300-1600 \mathrm{~s}^{-1}$ ). ${ }^{34}$ Additionally, our in vitro protocol has the advantage of removing a large part of the complications due to neural factors and humeral factors (isolated artery). Also by keeping the wall intact, our in vitro study takes into account the VSMC-extracellular matrix interactions which could be an important modulator of VSM tone. ${ }^{27}$

Third, we did not expose our arterial segment to a step increase but rather a ramp increase in pressure. However, there are studies ${ }^{31,32}$ demonstrating that this type of static myogenic response is not necessarily different from the response due to a step increase in pressure, at least as far as its final amplitude is concerned. In addition, the prompt response of the myogenic effect ${ }^{4}$ and the slow rate of pressure augmentation used in this 
study (around $170 \mathrm{~Pa} / \mathrm{s}$ or $1.3 \mathrm{~mm} \mathrm{Hg} / \mathrm{s}$ ) should allow us to use our pressure-diameter data to study the static myogenic response of the arterial segment.

Fourth, the hypertension model used in the study is different from the one commonly used in hypertension research (i.e., renal hypertension). The reason we chose the present model is that it produces an immediate step change in pressure, which from a biomechanical modeling point of view was optimal. Further, it allowed the study of very short adaptation to a step change in pressure. The study does not consider the role of angiotensin II on the VSM tone in presence of hypertension mainly because we focused entirely on the mechanical aspects without searching for the biological origin of the VSM tone alterations. It is likely that the renin-angiotensin system contributes to the observed VSM tone increase, without excluding other regulatory (or even intrinsic to VSM) mechanisms. Further studies are needed to answer this question.

Finally, the proposed model focuses on an acutely induced hypertension and any extrapolation of our results to hypertension in man should be made with caution.

\section{Simplified Model of VSM Tone}

There is good agreement between experimental data and the result of mathematical model proposed here [Figs. 3 and 4(B)]. Therefore, we suggest that this simple model captures well the characteristics of the evolution of stress-strain curves under normal and maximal VSM tone. In this model we assume that hypertension does not affect (a) the maximal active stress-strain curve and (b) the sensitivity of the myogenic response to strain rate (slope parameter $q$ ). These hypotheses, although plausible in the present situation, require further verification.

In summary, we observe that the carotid artery of Wistar rats under acute hypertension (days) develops enhanced basal and myogenic tones, the latter adaptation being principally achieved by an augmentation of the sensitivity of the myogenic response to the strain. Moreover, the enhanced basal and myogenic tone does not persist in long term (weeks) when the slower structural remodeling comes into play. In contrast with the response of resistance vessels, this transitory increase in the conduit artery VSM tone is beneficial from a biomechanical point of view because it leads to increased Windkessel effect and thus reduces heart load and systolic hypertension. Determination of the precise biological pathways governing the observed VSM adaptation to acute hypertension in conduit arteries will be an inevitable continuation of this work.

\section{ACKNOWLEDGMENTS}

The work presented in this paper is funded in part by the Swiss National Science Foundation (Grant No. 2100042321.94/2), the Swiss Federal Institute of Technology and by the Japanese Ministry of Education, Science and Culture [Monbusho International Scientific Research Program: Joint Research, No. 08044147 and Grant-in-Aid for Scientific Research (B) (2), No. 10480245].

\section{REFERENCES}

${ }^{1}$ Asano, M., K. Masuzawa-Ito, and T. Matsuda. Charybdotoxin-sensitive $\mathrm{K}+$ channels regulate the myogenic tone in the resting state of arteries from spontaneously hypertensive rats. Br. J. Pharmacol. 108:214-22, 1993.

${ }^{2}$ Asano, M., Y. Nomura, K. Ito, Y. Uyama, Y. Imaizumi, and M. Watanabe. Increased function of voltage-dependent $\mathrm{Ca}++$ channels and $\mathrm{Ca}(++)$ - activated $\mathrm{K}+$ channels in resting state of femoral arteries from spontaneously hypertensive rats at prehypertensive stage. J. Pharmacol. Exp. Ther. 275:775-783, 1995.

${ }^{3}$ Belik, J. The myogenic response of arterial vessels is increased in fetal pulmonary hypertension. Pediatr. Res. 37:196-201, 1995.

${ }^{4}$ Beven, J. A., J. L. Garcia-Roldan, and E. H. Joyce. Resistance artery tone is influenced independently by pressure and by flow. Blood Vessels 27:202-207, 1990.

${ }^{5}$ Cox, R. H. Alterations in active and passive mechanics of rat carotid artery with experimental hypertension. Am. J. Physiol. 237:H597-H605, 1979.

${ }^{6}$ Cox, R. H. Comparison of arterial wall mechanics in normotensive and spontaneously hypertensive rats. Am. J. Physiol. 237:H159-H167, 1979.

${ }^{7}$ Davis, M. J., and M. A. Hill. Signaling mechanisms underlying the vascular myogenic response. Physiol. Rev. 79:387423, 1999.

${ }^{8}$ Davis, M. J., and P. J. Sikes. Myogenic responses of isolated arterioles: test for a rate-sensitive mechanism. Am. J. Physiol. 259:H1890-H1900, 1990.

${ }^{9}$ Dunn, W. R., S. J. Wallis, and S. M. Gardiner. Remodeling and enhanced myogenic tone in cerebral resistance arteries isolated from genetically hypertensive Brattleboro rats. $J$. Vasc. Res. 35:18-26, 1998.

${ }^{10}$ Dzau, V. J., and G. H. Gibbons, Cell biology of vascular hypertrophy in systemic hypertension. Am. J. Cardiol. 62:30G-35G, 1988.

${ }^{11}$ Falcone, J. C., H. J. Granger, and G. A. Meininger. Enhanced myogenic activation in skeletal muscle arterioles from spontaneously hypertensive rats. Am. J. Physiol. 265:H1847H1855, 1993.

${ }^{12}$ Folkow, B. Comments on endpoints in hypertension: Peripheral resistance vessels - though mainly on their involvement as starting-points. Blood Press. Suppl. 2:34-38, 1997.

${ }^{13}$ Fridez, P., A. Makino, H. Miyasaki, J. Meister, K. Hayashi, and N. Stergiopulos. Short-term biomechanical adaptation of the rat carotid to acute hypertension: Contribution of smooth muscle. Ann. Biomed. Eng. 29:1-9, 2001.

${ }^{14}$ Fung, Y. Biomechanics. Motion, Flow, Stress and Growth. New York: Springer, 1990.

${ }^{15}$ Garcia, S. R., A. S. Izzard, A. M. Heagerty, and S. J. Bund. Myogenic tone in coronary arteries from spontaneously hy- 
pertensive rats. J. Vasc. Res. 34:109-116, 1997.

${ }^{16}$ Hansen-Smith, F., A. S. Greene, A. W. Cowley, Jr., L. Lougee, and J. H. Lombard. Structural alterations of microvascular smooth muscle cells in reduced renal mass hypertension. Hypertension 17:902-908, 1991.

${ }^{17}$ Hayoz, D., Y. Tardy, B. Rutschmann, J. P. Mignot, H. Achakri, F. Feihl, J. J. Meister, B. Waeber, and H. R. Brunner. Spontaneous diameter oscillations of the radial artery in humans. Am. J. Physiol. 264:H2080-H2084, 1993.

${ }^{18}$ Kuo, L., W. M. Chilian, and M. J. Davis. Coronary arteriolar myogenic response is independent of endothelium. Circ. Res. 66:860-866, 1990.

${ }^{19}$ Langile, B. Blood flow-induced remodeling of the artery wall. In: Flow-Dependent Regulation of Vascular Function, edited by J. A. Bevan and G. M. Rubanyi. New York: Oxford University Press, 1995.

${ }^{20}$ Laurent, S. Arterial wall hypertrophy and stiffness in essential hypertensive patients. Hypertension 26:355-362, 1995.

${ }^{21}$ Laurent, S., X. Girerd, J. J. Mourad, P. Lacolley, L. Beck, P. Boutouyrie, J. P. Mignot, and M. Safar. Elastic modulus of the radial artery wall material is not increased in patients with essential hypertension. Arterioscler. Thromb. 14:12231231, 1994.

${ }^{22}$ Lee, S., and G. W. Schmid-Schonbein. Biomechanical model for the myogenic response in the microcirculation: Part IIExperimental evaluation in rat cremaster muscle. J. Biomech. Eng. 118:152-157, 1996.

${ }^{23}$ Lee, K. M., K. Y. Tsai, N. Wang, and D. E. Ingber. Extracellular matrix and pulmonary hypertension: Control of vascular smooth muscle cell contractility. Am. J. Physiol. 274:H76-H82, 1998.

${ }^{24}$ Liu, S. Q., and Y. C. Fung. Relationship between hypertension, hypertrophy, and opening angle of zero-stress state of arteries following aortic constriction. J. Biomech. Eng. 111:325-335, 1989.

${ }^{25}$ Matsumoto, T., and K. Hayashi. Mechanical and dimensional adaptation of rat aorta to hypertension. J. Biomech. Eng. 116:278-283, 1994.

${ }^{26}$ Michel, J. B., A. Bardou, A. Tedgui, and B. Levy. Effect of descending thoracic aorta clamping and unclamping on phasic coronary blood flow. J. Surg. Res. 36:17-24, 1984.

${ }^{27}$ Mulvany, M. J., G. L. Baumbach, C. Aalkjaer, A. M. Heagerty, N. Korsgaard, E. L. Schiffrin, and D. D. Heistad, Vascular remodeling [letter]. Hypertension 28:505-506, 1996.

${ }^{28}$ Nurkiewicz, T. R., and M. A. Boegehold. High dietary salt alters arteriolar myogenic responsiveness in normotensive and hypertensive rats. Am. J. Physiol. 275:H2095-H2104, 1998.

${ }^{29}$ Osol, G., and W. Halpern. Myogenic properties of cerebral blood vessels from normotensive and hypertensive rats. Am. J. Physiol. 249:H914-H1215, 1985; Liu, S. Q., and Y. C. Fung. Relationship between hypertension, hypertrophy, and opening angle of zero-stress state of arteries following aortic constriction. J. Biomech. Eng. 111:325-335, 1989.

${ }^{30}$ Pohl, U., and R. Busse. Endothelium-dependent modulation of vascular tone and platelet function. Eur. Heart J. 11 B:3542, 1990.

${ }^{31}$ Schubert, R., and M. J. Mulvany. The myogenic response: established facts and attractive hypotheses. Clin. Sci. 96:313326, 1999.

${ }^{32}$ Stacy, D. L., and R. L. Prewitt. Effects of chronic hypertension and its reversal on arteries and arterioles. Circ. Res. 65:869-879, 1989.

${ }^{33}$ Westerhof, N., and M. F. O'Rourke. Haemodynamic basis for the development of left ventricular failure in systolic hypertension and for its logical therapy [editorial] [see comments]. J. Hypertens. 13:943-952, 1995.

${ }^{34}$ Zanchi, A., N. Stergiopulos, H. R. Brunner, and D. Hayoz. Differences in the mechanical properties of the rat carotid artery in vivo, in situ, and in vitro. Hypertension 32:180-185, 1998. 